1999-01-01

\title{
Some aspects of the adsorption of a Lennard-Jones gas on a rough surface
}

Douglas Henderson

Pawel Bryk

Stefan Sokolowski

Follow this and additional works at: https://scholarsarchive.byu.edu/facpub

Part of the Biochemistry Commons, and the Chemistry Commons

\section{Original Publication Citation}

Bryk, Pawe, Stefan Sokolowski, and Douglas Henderson."Some aspects of the adsorption of a Lennard-Jones gas on a rough surface." The Journal of Chemical Physics 11 (1999): 15-17.

\section{BYU ScholarsArchive Citation}

Henderson, Douglas; Bryk, Pawel; and Sokolowski, Stefan, "Some aspects of the adsorption of a LennardJones gas on a rough surface" (1999). Faculty Publications. 629.

https://scholarsarchive.byu.edu/facpub/629

This Peer-Reviewed Article is brought to you for free and open access by BYU ScholarsArchive. It has been accepted for inclusion in Faculty Publications by an authorized administrator of BYU ScholarsArchive. For more information, please contact ellen_amatangelo@byu.edu. 


\title{
Some aspects of the adsorption of a Lennard-Jones gas on a rough surface
}

\author{
Paweł Bryk and Stefan Sokołowski ${ }^{\mathrm{a})}$ \\ Department for the Modelling of Physico-Chemical Processes, Faculty of Chemistry, MCS University, \\ 20031 Lublin, Poland \\ Douglas Henderson \\ Department of Chemistry and Biochemistry, Brigham Young University, Provo, Utah 84602
}

(Received 31 August 1998; accepted 23 October 1998)

\begin{abstract}
We study the adsorption, including wetting, of a Lennard-Jones gas on a rough surface consisting of rough layer on a smooth substrate with which the gas interacts via a 9-3 potential. The rough layer is two molecular diameters thick and consists of a disordered quenched matrix of hard spheres. As well as interacting with the other matrix spheres by the hard-sphere potential, the matrix molecules interact with the gas molecules by the hard-sphere potential. Hence, on average, the degree to which the gas molecules can approach the substrate depends only on the density of the matrix layer. The density of this rough layer has a significant effect. As the density of the matrix layer increases, the adsorption isotherms pass from wetting to a prewetting transition, and, if the layer is dense enough, to partial wetting. It is interesting that the prewetting transition remains first order. (C) 1999 American Institute of Physics. [S0021-9606(99)71001-9]
\end{abstract}

Adsorption on a surface is a fascinating phenomenon. In recent years, there has been considerable interest in wetting. Of particular interest is the prewetting transition that is often a first-order transition. Evans and $\mathrm{Chan}^{1}$ have given a brief review of this phenomenon recently. Evans has also written a more detailed review. ${ }^{2}$

Most theories of wetting have been applied to the adsorption of a gas on a smooth surface. The effect of energetic and geometric heterogeneity on phase transitions, including those associated with wetting, involving flat surfaces and capillary condensation in pores has been studied. ${ }^{3-10}$ In these studies, the heterogeneity was introduced by mathematical constructions. Here, we wish to study these effects by means of a molecular model.

We consider the usual smooth surface that interacts with a gas via the 9-3 Lennard-Jones potential

$$
\boldsymbol{v}(z)=\boldsymbol{\epsilon}_{g s}\left[\left(\frac{\sigma_{g s}}{z}\right)^{9}-\left(\frac{\sigma_{g s}}{z}\right)^{3}\right] .
$$

The gas consists of Lennard-Jones molecules whose interaction potential is

$$
u(r)=\left\{\begin{array}{ll}
4 \epsilon\left[(\sigma / r)^{12}-(\sigma / r)^{6}\right] & r<r_{c} \\
0 & r>r_{c}
\end{array},\right.
$$

where $r_{c}$ is the cutoff distance. In our model, $\epsilon_{g s}=24 \epsilon$, $\sigma_{g s}=0.6 \sigma$, and $r_{c}=3 \sigma$. These parameters are representative of argon on graphite. Without loss of generality we assume $\sigma$ to be the unit of length, $\sigma=1$.

A layer consisting of a quenched matrix of hard spheres of diameter $\sigma_{h s}=\sigma$ is then placed on the "graphite" surface. The matrix molecules interact with each other and with the gas molecules by means of the hard-sphere potential. We

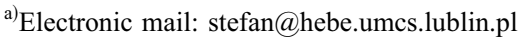

denote the matrix-matrix and the gas-matrix potentials by $u_{m m}(r)$ and $u_{g m}(r)$, respectively. The layer is $2 \sigma$ in thickness. The density of the matrix layer, $\rho_{m}(z)$, is a function of the distance from the surface. We assume the simplest form of this function, namely

$$
\rho_{m}(z)=\left\{\begin{array}{ll}
\rho_{m}^{0} & 0<z<2 \sigma \\
0 & \text { otherwise }
\end{array} .\right.
$$

Depending on $\rho_{m}^{0}$, the gas molecules partially penetrate the matrix layer (or do not penetrate at all, if $\rho_{m}^{0}$ is large enough). Of course, when $\rho_{m}^{0}=0$, there is no matrix layer present and the gas molecules can come right to the graphite surface.

To study the adsorption of the gas molecules by the surface, the inhomogeneous fluid density, $\rho(z)$, must be obtained. We start by writing the Born-Green-Yvon (BGY) equation for $\rho(z)$

$$
\begin{aligned}
\frac{\partial \ln \rho\left(z_{1}\right)}{\partial z_{1}}= & \beta \int d \mathbf{r}_{2} \rho\left(z_{2}\right) g\left(\mathbf{r}_{1}, \mathbf{r}_{2}\right) \frac{\partial u\left(\left|\mathbf{r}_{1}-\mathbf{r}_{2}\right|\right)}{\partial z_{2}} \\
& +\beta \int d \mathbf{r}_{2} \rho_{m}(z) g_{g m}\left(\mathbf{r}_{1}, \mathbf{r}_{2}\right) \frac{\partial u_{g m}\left(\left|\mathbf{r}_{1}-\mathbf{r}_{2}\right|\right)}{\partial z_{2}}
\end{aligned}
$$

where $g\left(\mathbf{r}_{1}, \mathbf{r}_{2}\right)$ and $g_{g m}\left(\mathbf{r}_{1}, \mathbf{r}_{2}\right)$ are the gas-gas and gasmatrix pair correlation functions. The BGY equation is an identity obtained from force balance considerations. This equation has been shown to be valid for quenched annealed mixtures. ${ }^{11}$ To proceed further, we need some approximation for the inhomogeneous pair functions, $g\left(\mathbf{r}_{1}, \mathbf{r}_{2}\right)$ and $g_{g m}\left(\mathbf{r}_{1}, \mathbf{r}_{2}\right)$. Of course, we could attempt to solve a set of equations for an inhomogeneous fluid and matrix. ${ }^{12,13}$ This would be difficult and time consuming. 


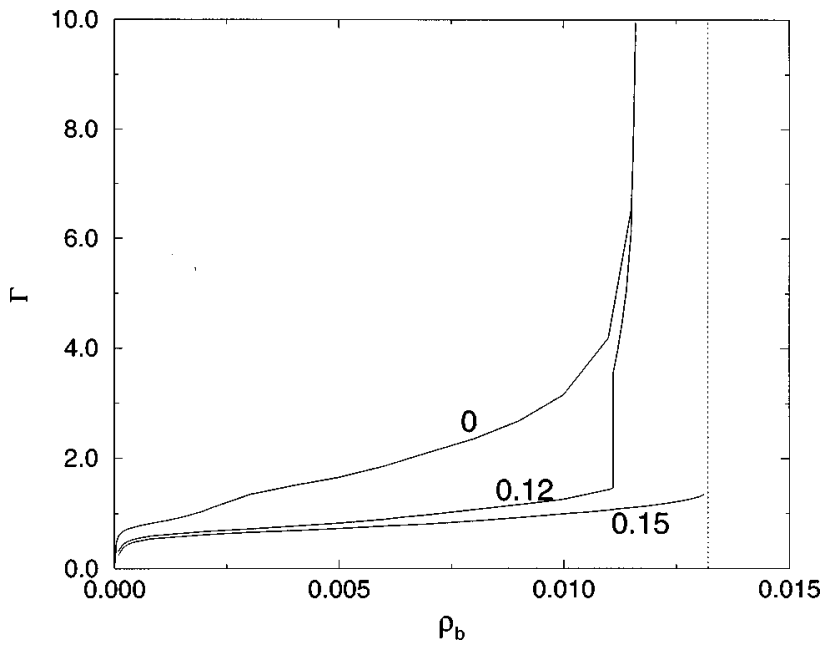

FIG. 1. Adsorption isotherms at $T^{*}=0.9$ for the matrix densities $0,0.12$, and 0.15 . The dotted vertical line gives the bulk vapor coexistence density.

In this study, we proceed in a simpler manner. We follow the Fischer and Methfessel (FM) ${ }^{14,15}$ theory, developed for single component nonuniform fluids, and next generalized to study adsorption of fluid mixtures. ${ }^{16}$ The BGY/FM approach yields results that agree well with simulations; also, it describes wetting. There is a close relation between this method and the density functional method ${ }^{15}$ but the BGY/FM method is simpler to implement. We can achieve a further simplification by using the Weeks-ChandlerAndersen (WCA $)^{17}$ prescription for dividing the gas-gas potential into a repulsive and an attractive tail, $u(r)=u_{\text {rep }}(r)$ $+u_{\text {att }}(r)$, with

$$
u_{\text {att }}(r)=\left\{\begin{array}{ll}
u\left(r_{\min }\right) & r<r_{\text {min }} \\
u(r) & r>r_{\text {min }}
\end{array} .\right.
$$

Recall that $r_{m}=2^{1 / 6} \sigma$. Again following WCA, we substitute the repulsive potential by the hard-sphere potential. Further, we replace $g\left(\mathbf{r}_{1}, \mathbf{r}_{2}\right)$ by the hard-sphere distribution function. To keep the analysis as simple as possible, we do not use an effective hard-sphere diameter but assume that $\sigma$ is a reasonable choice for the hard-sphere diameter.

In the WCA approach, the first integral in Eq. (4) splits into two terms. One involves an integral of the derivative of $u_{\text {att }}(r)$. We evaluate this integral in a mean-field approximation and replace the correlation function by unity. The second integral involves the derivative of the hard-sphere potential and can be cast in terms of the delta function. As a result, only the contact value of the pair correlation function is needed. The second integral in Eq. (4) involves only hardsphere correlation functions. Again only contact values are needed. We approximate the inhomogeneous correlation functions by those of a homogeneous fluid with an averaged density at the mid point of the two molecules in the inhomogeneous fluid. Thus, $g\left(\mathbf{r}_{1}, \mathbf{r}_{2} ; r_{12}=\sigma\right)=g^{0}\left(r_{12}=\sigma\right.$; $\widetilde{\rho}\left(z_{1}, z_{2}\right), \tilde{\rho}_{m}\left(z_{1}, z_{2}\right)$ and $g_{g m}\left(\mathbf{r}_{1}, \mathbf{r}_{2} ; r_{12}=\sigma\right)=g_{g m}^{0}\left(r_{12}=\sigma\right.$; $\left.\widetilde{\rho}\left(z_{1}, z_{2}\right), \tilde{\rho}_{m}\left(z_{1}, z_{2}\right)\right)$, where the superscript 0 denotes the homogeneous system. Following FM, we average $\rho(z)$ and $\rho_{m}(z)$ over a sphere of diameter $\sigma$ centered at $\mathbf{r}_{c}=\left(\mathbf{r}_{1}\right.$ $\left.+\mathbf{r}_{2}\right) / 2$. Thus,
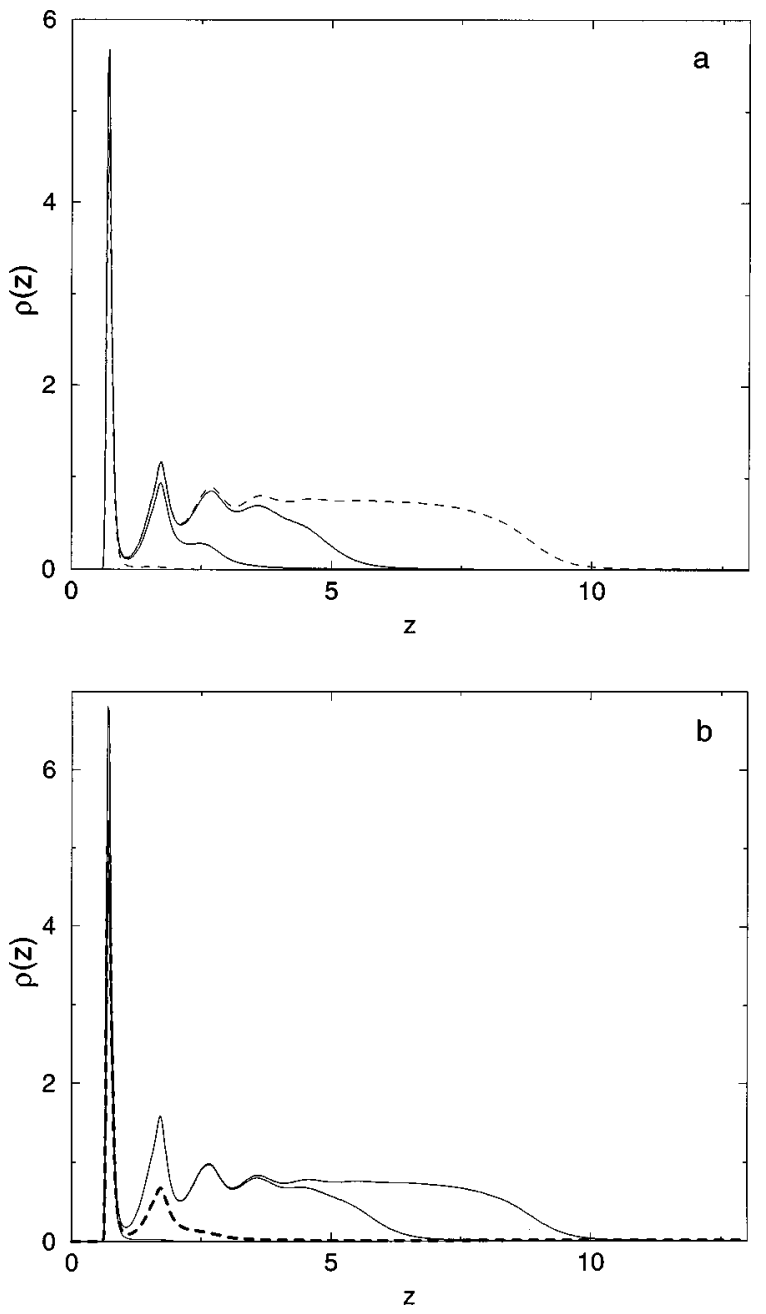

FIG. 2. Examples of the fluid density profiles at $T^{*}=0.9$. Part a is for $\rho_{m}^{0}$ $=0.12$. The solid lines give two coexisting profiles at $\rho_{b}=0.01105$; the dashed lines (from bottom to top) are for $\rho_{b}=0.001$ and 0.0113 , respectively. Part b is for $\rho_{m}^{0}=0$ (solid lines from bottom to top are for $\rho_{b}$ $=0.001,0.01$, and 0.001105 ) and for $\rho_{m}^{0}=0.15$ and $\rho_{b}=00132$ (dashed line).

$$
\widetilde{\rho}\left(z_{1}, z_{2}\right)=\tilde{\rho}\left(\frac{z_{1}+z_{2}}{2}\right)=\frac{1}{V} \int \rho\left(\mathbf{r}+\mathbf{r}_{c}\right) d \mathbf{r},
$$

where $V=\pi \sigma^{3} / 6$, with a similar equation for $\widetilde{\rho}_{m}\left(z_{1}, z_{2}\right)$. After integrating, our final equation is

$$
\begin{aligned}
\rho\left(z_{0}\right) / \rho_{b}= & \exp \left\{-\beta \int u_{\text {att }}\left(r_{02}\right)\left(\rho\left(z_{2}\right)-\rho_{b}\right) d \mathbf{r}_{2}+2 \pi \int_{z_{0}}^{\infty} d z_{1}\right. \\
& \times \int_{-\sigma}^{+\sigma} d z_{12}\left[\rho\left(z_{2}\right) g^{0}\left(\sigma ; \widetilde{\rho}\left(z_{1}, z_{2}\right), \tilde{\rho}_{m}\left(z_{1}, z_{2}\right)\right)\right. \\
& \left.\left.+\rho_{m}\left(z_{2}\right) g_{g m}^{0}\left(\sigma ; \widetilde{\rho}\left(z_{1}, z_{2}\right), \tilde{\rho}_{m}\left(z_{1}, z_{2}\right)\right)\right] z_{12}\right\},
\end{aligned}
$$

where $\rho_{b}$ is the bulk density of the gas.

The gas-gas and matrix-gas RDFs can be obtained in a variety of ways, including simulations. ${ }^{18,19}$ Simulations seem to be excessively sophisticated for the relatively simple arguments that have been used to obtain Eq. (7). In this work, we evaluate these functions by solving the replica Ornstein- 


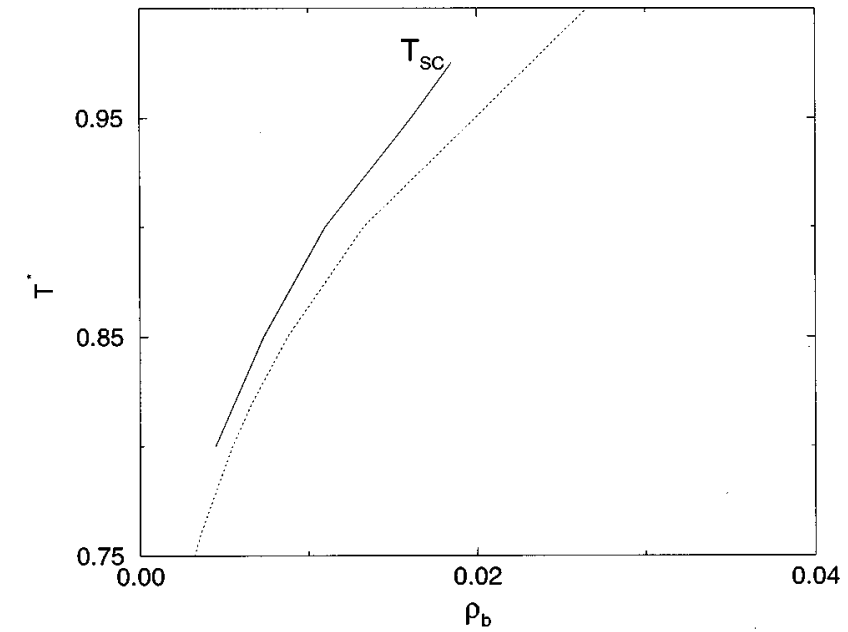

FIG. 3. Phase diagram. The dashed line is the bulk phase diagram, whereas solid line is the wetting line, terminating at the surface critical temperature, $T_{S C}$.

Zernike (ROZ) equations, using the Percus-Yevick (PY) approximation. The ROZ/PY approximation gives a good description of a homogeneous hard-sphere fluid in a disordered hard-sphere matrix consisting of hard spheres of the same size. We stress that recently similar theory has been successfully applied to study adsorption of hard spheres in slitlike with hard walls, filled with a hard-sphere matrix. ${ }^{20}$

As is seen in Fig. 1, where some adsorption isotherms, calculated from

$$
\Gamma=\int_{0}^{\infty} d z\left[\rho(z)-\rho_{b}\right],
$$

are plotted for the specific temperature $T^{*}=k T / \epsilon=0.9$. The isotherm for the matrix density $\rho_{m}^{0}=0$ is above the surface critical point whereas the isotherm for $\rho_{m}^{0}=0.15$ is clearly below the temperature at which prewetting first appears.

On the other hand, for values of $\rho_{m}^{0}$ in the vicinity of $\rho_{m}^{0}=0.12$, there is a clear first-order prewetting transition. The order of this transition is rather interesting. One might think that the rough disordered matrix layer would cause the transition, if it existed at all, to become continuous. We see from our results that this is not the case. The limiting value of $\rho_{b}=0.01325$ is the density of the vapor that is in equilibrium with the bulk liquid phase, calculated from the FM formalism. $^{21}$

In Fig. 2(a), density profiles are plotted for $\rho_{m}^{0}=0.12$. The existence of layers of two different thicknesses at $\rho_{b}$ $=0.011$ is clearly apparent. As $\rho_{b}$ is increased to the density of the vapor that coexists with the liquid phase, the adsorbed layer becomes infinitely thick.

Density profiles for $\rho_{m}^{0}=0$ are plotted in Fig. 2(b). It is clear that as $\rho_{b}$ is increased, there is a continuous growth in the thickness of the adsorbed "liquidlike" layer. As $\rho_{b}$ approaches the density of the vapor that coexists with the liquid phase, the adsorbed layer becomes infinitely thick and there is complete wetting. Also in Fig. 2(b), the density profile for $\rho_{m}^{0}=0.15$ is shown for the density $\rho_{b}=0.01320$ that is almost equal to the bulk coexistence density of $\rho_{b, \text { coex }}$ $=0.01325$. It is to be noted that this film remains thin.

The phase diagram of this system, obtained from this approach, is shown in Fig. 3 for the interesting case $\rho_{m}^{0}$ $=0.12$. Increasing $\rho_{m}^{0}$ shifts the line for prewetting to higher temperatures. The surface critical temperature for $\rho_{m}^{0}=0.12$ is $T_{S C}^{*}=0.97$. We have not yet located the exact value of the wetting temperature. For this value of $\rho_{m}^{0}$, it is between $T^{*}$ $=0.65$ and $T^{*}=0.70$.

We find that a rough disordered layer of hard spheres on a graphitelike substrate can change the adsorption isotherms significantly even if the density of the layer is small. For the single temperature that we have reported, we find that increasing the matrix density causes the adsorbed layer to pass from wetting to a first-order prewetting transition and then to partial wetting without a transition. Thus, increasing $\rho_{m}^{0}$ inhibits wetting. It is interesting that the prewetting transition remains first order.

It will be interesting to examine the effects on adsorption of changing the manner of formation and the nature of the constituents of the rough matrix layer. Such studies are being undertaken.

P.B. and S.S. are grateful to KBN for financial support under the Grant No. 3 T09 1111. The work of D.H. has been supported in part by the National Science Foundation (Grant No. CHE96-01971) and by the donors of the Petroleum Research Fund, administered by the American Chemical Society, Grant No. ACS-PRF3173-AC9.

${ }^{1}$ R. Evans and M. Chan, Phys. World 9, 48 (1996).

${ }^{2}$ R. Evans, in Fundamentals of Inhomogeneous Fluids, edited by D. Henderson (Dekker, New York, 1992), Chap. 3.

${ }^{3}$ M. Schoen and D. J. Diestler, Phys. Rev. E 56, 4427 (1997); Chem. Phys. Lett. 273, 296 (1997).

${ }^{4}$ M. Schoen and S. Dietrich, Phys. Rev. E 56, 499 (1997).

${ }^{5}$ G. Chmiel, L. Łajtar, S. Sokołowski, and A. Patrykiejew, J. Chem. Soc., Faraday Trans. 90, 1153 (1994).

${ }^{6}$ G. Chmiel, K. Karykowski, W. Rżysko, A. Patrykiejew, and S. Sokołowsk, Mol. Phys. 81, 691 (1994).

${ }^{7}$ G. Chmiel, A. Patrykiejew, W. Rżysko, and S. Sokołowski, Mol. Phys. 83, 19 (1994).

${ }^{8}$ G. Chmiel, A. Patrykiejew, W. Rżysko, and S. Sokołowski, Phys. Rev. B 48, 14454 (1993).

${ }^{9}$ D. Adelman, J.-F. Joanny, and M. O. Robbins, Europhys. Lett. 7, 731 (1988).

${ }^{10}$ J. L. D. Frok and F. van Swol, J. Phys. Chem. 108, 5588 (1998).

${ }^{11}$ W. Dong, E. Kierlik, and M. L. Rosinberg, Phys. Rev. E 50, 4750 (1994).

${ }^{12}$ O. Pizio and S. Sokołowski, Phys. Rev. E 56, R63 (1997).

${ }^{13}$ A. Kovalenko, D. Henderson, S. Sokołowski, and O. Pizio, Phys. Rev. E 57, 1824 (1998).

${ }^{14}$ J. Fischer and M. Methfessel, Phys. Rev. A 32, 2836 (1980).

${ }^{15}$ J. Fischer and U. Heinbuch, J. Chem. Phys. 88, 1909 (1988).

${ }^{16}$ S. Sokołowski and J. Fischer, Mol. Phys. 71, 393 (1990).

${ }^{17}$ J. D. Weeks, D. Chandler, and H. C. Andersen, J. Chem. Phys. 54, 5237 (1971).

${ }^{18}$ A. Given and G. Stell, J. Chem. Phys. 97, 4573 (1992).

${ }^{19}$ A. Meroni, D. Levesque, and J. J. Weis, J. Chem. Phys. 105, 1101 (1996).

${ }^{20}$ O. Pizio and S. Sokołowski, Phys. Rev. E 58, 2652 (1998).

${ }^{21}$ M. Wendland, Fluid Phase Equilibria 147, 105 (1998). 